\title{
Le voyage à Cubitas dans Sab de Gertrudis Gómez de Avellaneda
}

Paysages-traces, espaces de l'exhumation et paroles nouvelles d'une mémoire coloniale

Joséphine Marie

\section{(2) OpenEdition}

Journals

\section{Édition électronique}

URL : http://journals.openedition.org/agedor/1462

DOI : 10.4000/agedor. 1462

ISSN : 2104-3353

Éditeur

Laboratoire LISAA

Référence électronique

Joséphine Marie, «Le voyage à Cubitas dans Sab de Gertrudis Gómez de Avellaneda », L'Âge d’or [En ligne], 10 | 2017, mis en ligne le 14 janvier 2019, consulté le 06 mai 2019. URL : http://

journals.openedition.org/agedor/1462; DOI : 10.4000/agedor.1462

Ce document a été généré automatiquement le 6 mai 2019.

L'Âge d'or. Images dans le monde ibérique et ibéricoaméricain 


\title{
Le voyage à Cubitas dans Sab de Gertrudis Gómez de Avellaneda
}

\author{
Paysages-trace, espaces de l'exhumation et paroles nouvelles d'une \\ mémoire coloniale
}

Joséphine Marie

Face à une mémoire morcelée, tronquée, «lot d'obscurités » et "sentiment de chair discontinué ", d'après la formule des auteurs martiniquais de l'Éloge de la créolité ${ }^{1}$ (1990), Édouard Glissant rappelait préalablement, dans son Discours antillais (1981), le lien inextricable entre spatialités et temps caribéens. L'auteur témoigne : « Notre paysage est son propre monument: la trace qu'il signifie est repérable par-dessous. C'est tout histoire $»^{2}$. À notre sens, Sab (1841), le tout premier roman de la Cubaine Gertrudis Gómez de Avellaneda (Puerto Príncipe 1814 - Madrid 1873), révèle une conception et une exploitation poétique du territoire antillais qui font écho à ces réflexions - pourtant théorisées et appliquées plus d'un siècle après, dans un autre champ linguistique et culturel, celui des Antilles francophones. Si ce roman d'inspiration romantique est l'une des œuvres les plus connues de l'écrivaine, pour ne pas dire la plus connue, l'épisode particulier du voyage pour la contrée à la fois réelle et fictive de Cubitas, centre névralgique du récit, a finalement été peu étudié sous cet angle.

Dans cette fiction, les sites et les communautés reconstitués par l'écrivaine native proposent une topographie distinctive qui se dresse face à l'Europe. Ils constituent une première appropriation et représentation des territoires colonisés, par un auteur qui entend les présenter au lectorat espagnol tout en les valorisant (dans les fictions avellanédines les paysages américains sont, par exemple, plus propices à l'expérience sublime que les paysages européens). À l'instar des lieux évoqués et décrits sur le mode de l'esthétique du sublime, du pittoresque ou encore du discours de l'ethnologue, leur construction peut répondre à un désir d'ancrage. Dans ce sens, leur place dans la diégèse révèle que le récit, en mettant la description en son centre, ne rompt pas avec cette tradition ${ }^{3}$.

Mais ces figures paysagères ne sont qu'un des éléments d'une dialectique qui nous mène de l'estampe du lieu, point fixe par antonomase, vers divers espaces mouvants dont les 
frontières sont esquissées nouvellement ${ }^{4}$. Si Cubitas, localité située dans la région de Camagüey, renvoie bien à un référent réel, l'auteur la réélabore toutefois en la soumettant à sa fantaisie, au fil de l'avancée d'une nouvelle conscience historique. Le récit ne se contente alors pas $d^{\prime}$ '« organiser " ${ }^{5}$ des lieux, il les désorganise tout à fait. Gómez de Avellaneda se serait d'ailleurs inspirée d'une visite de la grotte de María Teresa (sierra de Cubitas), dont le compte rendu, qu'elle aurait commandé de Séville, aurait été publié dans les Memorias de Real Sociedad de los Amigos del País de 1839. Il est intéressant de constater que l'attention qu'elle a portée à cet endroit, particulièrement lié à la présence aborigène dans la région de Puerto Príncipe, et qui, à l'époque, fait l'objet d'explorations et d'interrogations sur cette présence, reposerait donc sur l'exploitation et le remaniement de sources provenant d'Espagne pour une réécriture des Amériques.

Devenant monde imaginaire qui délaisse la perspective laudative et/ou mimétique, pour privilégier autrement le symbole, Cubitas permet ainsi d'établir des variétés inédites des discours de la mémoire. Les espaces ne se limitent plus au désir d'édification et de mise en valeur traditionnelle du territoire. Conjointement à l'émergence du logos, ils perdent progressivement leurs habituelles fonctions de représentation, pour nous proposer la vision de multiples cheminements, au sein de sites palimpsestes et de paysages-traces ${ }^{6}$. Il s'agit alors de comprendre comment, au-delà de la matérialité d'un lieu, d'un point défini, c'est précisément cette refonte des espaces qui ouvre la voie à une contre-histoire par le paysage, contre-point de l'Histoire officielle de la colonisation, de l'Histoire des "vainqueurs », dans le contexte nouveau de la perte des colonies.

\section{Mouvances des formes paysagères et autres motifs : des strates d'histoire}

5 C'est au chapitre Ix de la première partie que les protagonistes (le colon, sa famille et leur esclave Sab), c'est-à-dire les représentants d'une grande part de l'histoire du peuplement antillais, entreprennent ce voyage qui s'apparente à un retour vers un espace originel occulté, si l'on en croit l'épigraphe suivante, qui oriente la lecture dudit chapitre, et qui n'est autre qu'une citation du poète cubain Heredia, figure toujours fondatrice dans l'œuvre avellanédine: "Do fue la raza candorosa y pura / que las Antillas habitó ?- La hiere / del vencedor el hierro furibundo,/ tiembla, gime, perece / y como niebla al sol desaparece." C'est précisément à Cubitas que vit le personnage de la vieille Indienne Martina qui prétend descendre du légendaire roi Camagüey. Nous sommes apparemment encore dans le mythe d'une pureté originelle, avec ces mots liminaires qui ne sont pas ceux de Gómez de Avellaneda, mais nous répondons également à une première invitation à reconsidérer l'Histoire.

\section{Le prisme du fantastique}

6 Cubitas, zone géographique à la périphérie du cadre principal, se situe également en marge de la narration, si l'on considère les modalités du traitement du lieu, ici fortement "spatialisé », à la différence du reste du récit. Sur ce point, c'est toute l'esthétique présidant à la construction de la zone qui en fait, a priori, un élément marginal de l'histoire. En effet, Cubitas a tout de l'espace fantastique ponctuellement intercalé dans une diégèse qui, dans son ensemble, ne révèle pas le choix de ce mode d'appréhension du réel. En introduisant ce nouvel espace, la narration met fin à la présence du locus amoenus 
et du lieu propice à l'expérience sublime que représentent l'habitation principale de Bellavista et de ses alentours. C'est explicitement que la voix narrative signale la variation fantastique dans ce nouvel univers :

La noche se acercaba mientras tanto, y sus pardas sombras robaban progresivamente a los viajeros los paisajes campestres que les rodeaban. La rica vegetación no ofrecía ya sus variadas tintas de verdura y las colinas lejanas presentábanse a la vista como grandes masas de sombras. A medida que se aproximaban de Cubitas el aspecto de la naturaleza era más sombrío: bien pronto desapareció casi del todo la vigorosa y variada vegetación de la tierra prieta, y la roja no ofreció más que esparramados yuraguanos, y algún ingrato jagüey que parecían en la noche figuras caprichosas de un mundo fantástico. El cielo empero era más hermoso en estos lugares: tachonábase por grados de innumerables estrellas, y cual otro ejército de estrellas errantes poblábase el aire de fúlgidos cocuyos, admirables luciérnagas de los climas tropicales. Carlota detuvo de repente su caballo e hizo observar al mulato una luz vacilante y pálida que oscilaba a lo lejos en lo más alto de la empinada loma. ${ }^{8}$

Le nouveau paysage de Cubitas est inconnu et changeant, et en cela réside la part de son inquiétante étrangeté : les ombres, dont celles des collines, s'étendent progressivement, la végétation change et s'appauvrit, la nature dans son ensemble se fait plus sombre, les lumières oscillent et la terre elle-même change de nature.

Le recours au genre fantastique peut d'abord être lu comme une stratégie de contournement adoptée par l'écrivaine. Outre ce que nous allons dégager dans ce travail qui relie le traitement de ce paysage avellanédin aux poétiques postcoloniales des ères caribéennes et hispano-américaines, de manière générale, la pratique du genre fantastique, genre privilégié par les romantiques, n'est pas sans lien avec l'aspiration de ces derniers à une forme de savoir total. Ce savoir révèle, non pas l'adéquation de l'homme au monde, mais son étrangeté, ainsi que le caractère tragique de son âme. Le fantastique, en particulier à travers la fuite dans le rêve, constituait déjà un mode d'expression permettant cette exploration du Moi et de son inconscient ${ }^{9}$. Cette pratique est également à relier à l'émergence et au développement d'une production romanesque féminine au XIX ${ }^{\mathrm{e}}$, notamment en Amérique latine (on trouve par exemple, pour ne nommer qu'eux, les écrits de Juana Manuela Gorriti). Le « détour » fantastique constituait ainsi un cadre favorable à l'expression indirecte, car apparemment placé hors du champ du «réel», d'un discours critique sur la condition féminine, ou sur les événements historiques d'une Conquête problématique. Il permet d'introduire une relecture des faits relativement subversive, dans un contexte qui rendait difficile, voire impossible, la publication d'une expression plus directement reliée au référent réel. Cela n'a d'ailleurs pas suffi à empêcher la censure du roman qui, en 1844, n'a pu entrer sur le territoire cubaiin $^{10}$.

9 Tout en répondant à la nécessité d'adopter cette stratégie, l'esthétique est consubstantielle à une dynamique de mise en mouvement générale. Elle se met en place, émerge depuis la présentation d'un « chemin » à parcourir pour atteindre une destination finale. Les alentours et le centre de Cubitas, village d'une Martina dépositaire de ses légendes, mystérieuse et inquiétante destination, dissonante face au traditionnel lieu paradisiaque, deviennent en effet le lieu presque inaccessible d'une histoire, dont on nous signifie avant tout qu'elle a été cachée, et à laquelle il s'agit précisément d'accéder depuis une autre logique. C'est ainsi que la vision marginale, l'endroit de la périphérie devient le centre. Dans cette perspective, soulignons d'ailleurs la place symbolique, car centrale, du 
chapitre IX. L'œuvre en comprend effectivement 16, auxquels s'ajoute une conclusion, soit 17 fragments.

Au-delà de l'enjeu de la création d'un lieu voulant matérialiser l'origine et relevant du mythe, c'est bien l'expédition, la traversée d'un espace mémoriel qui importe. Rappelons que les personnages n'arrivent à destination qu'à la fin du premier des trois chapitres de l'épisode. Leur voyage, difficile déplacement au fur et à mesure que la nuit tombe, devient métaphore du dévoilement, de l'avancée d'une conscience. Visiblement, la nature ne répond qu'à sa propre fantaisie, laquelle semble échapper à l'homme, qui la contemple mais ne la domine pas. Malgré ces mutations et l'aspect fugace ou indiscernable de certains des composants de l'impossible tableau d'une matière qui se dérobe, "figuras caprichosas de un mundo fantástico" (rappelons l'emploi de ce substantif polysémique au pluriel, pour désigner une variété de formes sans véritablement les déterminer), le paysage garde d'abord toutefois une certaine forme de matérialité. Celle-ci repose sur quelques éléments singuliers qui le constituent et s'enracinent en lui, dans ce qui relève finalement de la prosopopée.

\section{Symbolique de la terre rouge et des arbres pliés (palmier-balais et ficus)}

11 Vient d'abord la terre rouge. Les variations de la couleur de la terre nous mènent immédiatement à la percevoir comme un élément porteur de traces. D’une terre foncée, donc caractérisée par une opacité, qui fait qu'il est impossible de lire en elle, nous passons à un sol dont, par contraste, la vivacité du rouge, couleur du sang, apparait comme un signe visible et facilement déchiffrable. Avec les arbres, cette dimension symbolique du jeu chromatique, en écho avec les autres éléments, se fera encore plus évidente. En effet, font également partie du paysage des "yuraguanos" et des "jagüeyes"11, arbres dont les spécificités sont respectivement soulignées par Gómez de Avellaneda, dans des notes paratextuelles :

El Yuraguano es un arbusto de la familia de los guanos con muchas hojas parecidas algún tanto a las de la palma: aquellos a que se hace referencia en esta historia, y que abundan en las inmediaciones de Cubitas, son más altos que los yuraguanos comunes. No crece este arbusto recto y airoso como la palma, antes por el contrario su tronco se tuerce por lo regular, y a veces se tiende casi horizontalmente.

El Jagüey al principio no es más que un bejuco que se enreda a un árbol. Crece prodigiosamente; cubre y oprime con sus ramas el tronco que le ha sostenido y acaba por secarle. Entonces conviértese él en árbol corpulento; y la multitud de sus ramas que tiende de una manera caprichosa, sus raíces gruesas y visibles sobre la superficie de la tierra y las desigualdades de su tronco le dan un aspecto particular.

12 En faisant l'objet d'une glose supplémentaire qui les isole et met en évidence leurs caractéristiques, ces arbres sont dotés d'une plus grande valeur que celle que l'on attribue aux autres pièces du paysage. Ils font figure d'exception remarquable. Ils constituent une forme supplémentaire des variations déclinées tout au long de l'épisode, et à divers niveaux (isotopie du mouvement et du changement, variantes esthétiques, écart géographique). La voix narrative les érige manifestement comme des monuments qu'on ne saurait ignorer.

13 Les définitions proposées par l'auteure sont donc des définitions «négatives ». Dans le texte, l'élément naturel accède à l'existence dans la négation et la privation. Les premiers arbres ne se dressent pas comme le palmier, car leur tronc se tord, ce qui les plie parfois presque horizontalement. Quant au second motif, on nous dit qu'il s'agit d'une liane qui 
s'enroule autour de l'arbre, croît rapidement jusqu'à le couvrir, à opprimer le tronc qui l'a soutenue, et finit par l'assécher. La charge de négativité que comporte l'émergence dudit végétal, en particulier le second, alors présenté comme un parasite dont les racines volumineuses ne parviennent pas à s'ancrer tout à fait dans la terre, renvoie ici à la destruction de l'arbre qui lui sert de support. Il ne s'agit plus de signifier le difficile accès à l'existence du marqueur symbolique lui-même, mais de mettre en lumière une sorte d'ersatz. Celui-ci entend se substituer à la vie originelle. Il est porteur de mort.

Cette précision invite à entendre que, dans sa forme, le paysage est bel et bien soumis à un processus de recréation symbolique au service des discours sur l'Histoire, excluant définitivement la primauté de sa conformité avec la réalité physique. Malgré la caution prétendument scientifique que l'usage de la note cherche apparemment à conférer au roman, là n'est pas son véritable objet, ni son véritable intérêt. Ces arbres intégrés dans le sol de la terre rouge sont les premiers à apparaître à Cubitas. Sang, pliure ou courbure qui attestent de l'impossible droiture de la croissance, spoliation de la substance vitale et de l'espace de développement, sont autant de phénomènes immédiatement incarnés dans cette terre. Signes naturels, stigmates qui suggèrent l'existence d'une histoire en la matérialisant, ils font l'économie du concept. Ils marquent la préférence pour la forme symbolique tangible, visant à signifier une première fois, cherchant à fournir une première intuition à la conscience des personnages et du lecteur.

\section{L'obscurité lumineuse du chemin, de la grotte et de la cabane}

15 Une fois parvenus à Cubitas, les personnages poursuivent leur cheminement dans les méandres des grottes, puis dans la choza de Martina elle-même.

16 La grotte, lieu immémorial, constitue le deuxième espace signifiant de ce monde fantastique. Sa construction procède des mêmes ressorts que le chemin, avec lequel elle partage la fonction de paysage-trace. Nous immergeant, non plus dans l'histoire de la destruction conquérante, mais dans celle d'une époque antérieure, elle fait surgir des pans de la vie indienne qui marquent ainsi une avancée supplémentaire du voyage spatiotemporel. Elle présente également des objets dont on souligne encore la singularité et la rareté. À l'instar des pièces paysagères du chemin, ces objets apparaissent comme autant de marqueurs qui rappellent un passé lointain et font émerger la vision suivante :

Son notables entre estas salas la de la Bóveda por su capacidad y la del Horno cuya entrada es una tronera a flor de tierra por la que nos se puede pasar sino muy trabajosamente y casi arrastrándose contra el suelo. Sin embargo, es de las más notables salas de aquel vasto subterráneo y las incomodidades que se experimentan, al penetrar en ella, son ventajosamente compensadas con el placer de admirar las bellezas que contiene. Deslúmbrase el viajero que al levantar los ojos, en aquel reducido y tenebroso recinto, ve brillar sobre su cabeza un rico dosel de plata sembrado de zafiros y brillantes, que tal parece en la oscuridad de la gruta el techo singular que la cubre. Empero, pocos minutos puede gozarse impunemente de aquel bello capricho de la naturaleza, pues la falta de aire obliga a los visitadores de la gruta a arrojarse fuera, temiendo ser sofocados por el calor excesivo que hay en ella. El alabastro no supera en blancura y belleza a las piedras admirables de que aquellas grutas, por decirlo así, se hallan entapizadas. El agua, filtrando por innumerables e imperceptibles grietas, ha formado bellísimas figuras al petrificarse. Aquí una larga hilera de columnas parecen decorar el peristilo de algún palacio subterráneo; allá una hermosa cabeza atrae y fija las miradas: en otra parte se ven infinitas petrificaciones sin formas determinadas, que presentan masas de deslumbrante blancura y figuras raras y caprichosas. Los naturales hacen brotar en 
la cueva llamada de María Teresa pinturas bizarras designadas en las paredes con tinte de vivísimos e imborrables colores, que aseguran ser obra de los indios, y mil tradiciones maravillosas prestan cierto encanto a aquellos subterráneos desconocidos; que realizando las fabulosas descripciones de los poetas recuerdan los misteriosos placeres de las hadas. Nadie ha osado todavía penetrar más allá de la undécima sala. Se dice, empero, vulgarmente que un río de sangre marca su término visible, y que los abismos que le siguen son las enormes bocas del infierno. La ardiente imaginación de aquel pueblo ha adoptado con tal convicción esta extravagante opinión que, por cuanto hay en el mundo, no se atreverían a penetrar más allá de los límites a que se han concretado hasta el presente los visitadores de las cuevas, y lo estrecha y peligrosa que se va haciendo la senda subterránea, a medida que se interna, parece justificar sus temores. ${ }^{13}$

17 Non sans faire écho au paysage entourant le chemin qui mène à Cubitas, la salle "del Horno" laisse ainsi voir de capricieuses figures, à la forme également indéterminée et étrange. À leur tour, celles-ci feront écho aux motifs de la grotte María Teresa, dont l'évocation se conclut sur une vision d'ensemble qui réunit tous les éléments au sein d'un même monde de traditions explicitement qualifiées de "merveilleuses". Le monde souterrain entre alors en conformité avec les descriptions «fabuleuses » du monde des « fées ». Les motifs de la dernière grotte à laquelle on peut accéder (mais l'espace, lui, est présenté comme infini, il n'a pas de limite) ne sont autres que des peintures rupestres ancestrales, qu'on ne peut ignorer du fait de la vivacité de leurs coloris, et qui se donnent à voir comme autant de traces indélébiles de l'existence d'une communauté éteinte. L'abolition de la linéarité de l'histoire et du cloisonnement des périodes sur un axe passé, présent, futur, est ici assurée par l'incertitude. Encore aujourd'hui, nous dit-on, on ignore la nature du dernier endroit de cet ensemble atemporel. C'est donc en traversant un ensemble non borné de grottes sans âge, apparemment vieilles comme le monde, que futur et certitude pourront éventuellement émerger.

18 Si la visite des grottes constitue une indéniable exploitation de l'allégorie platonicienne de la caverne, la description du chemin annonçait déjà que la lumineuse métaphore nocturne allait être filée tout au long du texte avellanédin, lorsque celui-ci s'ancre à Cubitas. Sur le chemin, la lumière constitue un véritable motif de la transition, au sens propre, dans l'économie du texte, comme au sens figuré, dans la progression aussi bien géographique que cognitive. Les foyers lumineux qui apparaissent çà et là (étoiles, lucioles buvant le jus de la canne dans les plantations et dont se servaient les Indiens pour s'éclairer, et la mystérieuse lumière fugace émergeant du haut de la colline), sont tous des symboles d'un nouvel éclairage porté sur le monde, d'une vision nouvelle et pourtant ancestrale. La lumière de la colline qui représente l'âme du roi Camagüey resté sans sépulture, fera émerger le récit légendaire dont $\mathrm{Sab}$ est la voix. Si, là encore le paysage témoigne, il «met en lumière », il « dévoile » un crime originel, avant que le récit de la parole humaine ne l'explicite. À cet égard, dans tous les espaces dressés, la lumière marque le passage. Elle est la métaphore d'un dévoilement qui se construit sur l'isotopie du clair/obscur présentant mille variations et donnant toute sa portée symbolique à la mise en mouvement initialement dégagée.

$19 \mathrm{Si}$, pour les Romantiques, l'exploitation de l'imagination nocturne et les jeux de clair/ obscur présupposent cette affinité entre l'imagination et la part nocturne du monde et du $\mathrm{Moi}^{14}$, nous verrons comment, dans l'œuvre de Gómez de Avellaneda, elle est également à relier à cet enjeu de l'exhumation et de la mise en scène de la mémoire des terres antillaises colonisées. L'élément nocturne y est décisif et central. Il constitue un exemple représentatif de l'articulation entre les visions romantiques du monde et les quêtes 
esthétiques et idéologiques propres aux champs antillais et hispano-américain, qui entendent s'extirper des problématiques universelles/universalisantes et d'un certain européocentrisme. Le contenu symbolique est double. La nuit romantique se lie à la complexité fondamentale de l'homme et de son imagination, vision déjà héritée des Lumières, alors que la nuit « antillaise » se lie à la particularité du monde de la plantation, de son histoire. Le texte cherche à intégrer et à symboliser un rapport à l'espace-temps qui ne serait pas seulement lié aux cosmovisions ayant émergé en Europe. Le nocturne constitue alors un nouvel espace de subversion et renvoie à un autre rapport à la parole et à la temporalité, comme nous le verrons.

Enfin, la dernière étape de l'expédition est la sombre maison de Martina, origine et foyer de l'instance représentant la parole mémorielle. À l'instar de la grotte, le faible éclairage permet également de souligner des éléments naturellement lumineux ou ponctuellement éclairés, pour des effets de contrastes toujours significatifs.

21 Ces espaces s'éloignent donc de la représentation binaire et antithétique du locus amoenus /locus horribilis, lesquels sont caractéristiques du sublime et du pittoresque romantiques. C'est précisément parce qu'ils sont gages de cette plaisante ou saisissante nouveauté, acquise au prix d'efforts, qu'ils s'extirpent d'une vision exclusivement duelle. Certes, le chemin est inquiétant, la maison de Martina se présente comme un lieu peu accueillant, et les dangereuses grottes, difficiles d'accès, ne sont pas sans s'apparenter (toutefois selon la rumeur, c'est-à-dire sans certitude) aux bouches de l'enfer. Le nom même de la salle "del Horno" annonce les caractéristiques d'une antichambre des Enfers. Mais chaque description fait clairement entendre que l'ensemble est toujours chargé de la promesse du ravissement des yeux et peut donner lieu à la découverte de trésors. Dans cette reprise de l'allégorie de la caverne déclinée sous trois formes, les descriptions associent explicitement effort, plaisir de la vue et dévoilement.

\section{D'une « petite » Cuba à l'autre : dissolution du lieu, démultiplication des espaces}

L'ultime destination qu'est la maison de Martina aurait dû nous mener vers une fixité et une capacité de «localisation » plus certaine. Or il n'en est rien. Après la grotte qui s'étend à l'infini et se perd dans cette nouvelle mise en espace, la choza (proche de la ruine) n'est pas davantage un lieu d'ancrage. Réduite à peu de motifs, il est difficile de se la représenter dans son existence physique et matérielle. Elle tend même à se dissoudre par de multiples biais qui font d'elle un espace toujours transitoire.

Elle est d'abord présentée comme la substitution d'un premier lieu, lequel n'est envisagé que depuis sa destruction ("Un incendio consumió mi casa", "el fatal incendio que me redujo a la indigencia", "El incendio consumía mi morada y yo [...] veía los rápidos progresos del fuego" ${ }^{15}$ ). En d'autres termes, elle est d'entrée de jeu envisagée comme un lieu qui n'est plus. Ces allusions à l'incendie d'une première demeure sont révélatrices $\mathrm{du}$ souhait de mise en scène de la décomposition de l'habitat que l'auditoire, comme le lecteur, peuvent alors visualiser. Dans le récit ensuite prospectif, à partir d'une réplique de don Carlos (réplique qui permettra au passage de souligner la bonté paternaliste de ce dernier), le lecteur pourra encore visualiser un nouveau remplacement de la cabane par une autre habitation ("la mejor de mis estancias"16).

24 Le motif du bois, ici rappelé à loisir et décliné sous diverses formes, fait de la maison de Martina un lieu «mal fini », mal formé, difficile à discerner. Il apparaît également comme un lieu plein d'aspérités qui ne disent pas la fixité de la matière lisse, mais plutôt le 
mouvement, les mutations. Par association avec l'image de l'habitation précédente en flammes, le bois signifie également la potentielle décomposition d'un lieu qu'il compose pourtant. Morcelé par ces quelques motifs disjoints, l'ensemble, lorsqu'il est évoqué, est d'ailleurs toujours soumis à une réduction hyperbolique de sa dimension, et l'obscurité qui lui est inhérente, achève de le dissoudre. Le lieu misérable est donc à peine décrit et potentiellement décomposé. Enveloppé, non par la matérialité, mais par la virtualité, il est traversé par un mouvement qui le balance entre deux, voire trois, temporalités (le passé de la cabane incendiée, le site relatif au présent des personnages et le futur lieu de vie) et qui l'empêche de se fixer.

Le récit finit de mettre en scène la dissolution esthétique du lieu, lorsqu'il est associé au personnage marginal. D'une part, la maison de Martina réapparaît indirectement, non dans le déroulement de l'action d'une diégèse déjà ancrée dans le passé, mais dans le récit rétrospectif que constitue la lettre de Sab, intercalée dans la narration ("He venido a morir cerca de mi madre y de mi hermano"17). Associé à un passé du passé, à un récit dans le récit, cet espace, encore moins que la grotte ou le chemin, ne saurait assumer la fonction de véritable cadre de l'action. D'autre part, la dernière zone de l'action romanesque sera non Bellavista mais Cubitas, qui revêt ici la forme absolue du non-lieu ${ }^{18}$ avec la tombe de Sab. Celle-ci émergera exclusivement au travers de la métonymie de la croix en bois, placée au centre de la mise en scène d'une sépulture visitée par la fantomatique Indienne ${ }^{19}$.

Notons d'ailleurs que cet aspect contraste tout à fait avec l'essence que la narration confère au personnage de Martina, au contraire inscrite et enracinée dans une forme de pérennité ou d'atemporalité. Si don Carlos de B affirme qu'il la trouve toujours inchangée, elle se présente elle-même comme le "vieil arbre de la montagne $»^{20}$ sur lequel le temps n'a aucun effet. Un tel contraste est d'autant plus perceptible que cette permanence de l'être, en opposition à celle du lieu, est signifiée par l'allégorie de l'arbre, c'est-à-dire par une exploitation nouvelle mais inverse du motif du bois. Cette figure verticale permet de compléter l'orientation établie par l'horizontalité du mouvement des voyageurs. La figure martinienne est érigée au cœur d'une nature en mouvement, seule vision possible de son éventuel ancrage. La proximité géographique entre la maison et les grottes, déjà annoncée par $\mathrm{Sab}^{21}$, devient assimilation et spatialisation du paysage absolue, lorsque l'on sait que Martina, suite à l'incendie de sa maison, dit s'être réfugiée dans ces mêmes grottes ("no hallé otro asilo que esas cuevas"22).

En ce sens, la toponymie de Cubitas - en proposant une reprise du nom de l'Ile avec l'ajout d'une suffixation diminutive, ainsi que l'ajout de ce que l'on peut entendre comme la marque d'un pluriel - est fortement suggestive. L'espace divisé en trois zones symboliques que sont le chemin, les grottes et la maison de Martina (chacune entretenant un rapport métonymique à l'ensemble) se constitue donc d'une association de «petites Cuba » à traverser. L'addition de ces trois "cubitas» ne forme pas un ensemble géographique à l'image du référent « réel». Chacune est un univers qui fait écho aux autres, dans une continuité de la variation, pour toujours rappeler l'histoire insulaire. Depuis cette perspective, Cubitas peut à son tour être lu comme une synecdoque du territoire cubain. L'ensemble devient ainsi lui-même un espace désignant le tout de l'ile, et plus largement les terres antillaises, soumises à certains processus historiques communs.

28 Traversée par des déplacements, la vision apparemment morcelée de Cubitas acquiert donc une première forme d'unité par la dis-continuité spatiale, avec des non lieux qui ne 
sont plus uniques, mais fondus et démultipliés dans un continuum où les temporalités se multiplient également. Le rappel des charges symboliques qui provoquent les mêmes effets de sens particuliers et convergents, sont là pour révéler une appréhension de l'espace et un rapport au temps non strictement linéaire. Les variations inscrivent immédiatement les lieux dans une juxtaposition de couches temporelles qui les dissout puisqu'elle rend impossible leur fixité et leur permanence. Ces espaces-temps sont tout autant ceux des origines, du présent et du futur. À l'instar de certaines poétiques postromantiques des espaces antillais, le paysage imaginé est effectivement entendu et reconstruit comme trace d'une temporalité discontinue au sein de laquelle la datation est impossible ${ }^{23}$. La contre-poétique d'É. Glissant ne dit pas autre chose, lorsqu'elle évoque un calendrier qui ne se construit "qu'à la mesure des calamités naturelles» et qui ne supporte « nulle linéarité » :

[...] ainsi le temps se retourne en nous. [...] un vécu de l'histoire, à quoi nous introduisent le combat sans témoins, l'impossibilité même de la datation même inconsciente, conséquence du raturage de la mémoire en tous. Car l'histoire n'est pas seulement pour nous une absence, c'est un vertige. Ce temps, que nous n'avons jamais eu, il nous faut le reconquérir. Nous ne le voyons pas s'étirer dans notre passé et nous porter tranquilles vers demain, mais faire irruption en nous par blocs, charroyés dans des zones d'absence où nous devons difficilement, douloureusement, tout recomposer. ${ }^{24}$

\section{Logos et voix du paysage : l'ambivalente polyphonie pour l'avancée d'une conscience historique}

Cubitas constitue également une refonte de la représentation car, sous le lieu "réel », se niche l'espace des récits, l'espace des poètes des grottes, davantage virtualité de la parole qu'effectivité géographique. On peut ainsi voir dans les dessins des grottes quelque chose qui rappelle la fonction des codex précolombiens : un espace graphique qui sert d'appui au récit, qui «motive » la parole, une parole qui met l'espace en mouvement. Également présenté comme espace de sépulture (des figures finalement fantomatiques de Camagüey, de $\mathrm{Sab}^{25}$ et de Martina), Cubitas est définitivement associé à l'Indien(ne) et à l'esclave, tous deux porteurs d'histoires, héraults du récit.

La symbolique spatiale peut donc pallier une mémoire lacunaire parce qu'elle est fusion de la terre, de la chair et de la voix. Nous l'avons vu, le paysage du chemin comme la matérialité des pierres de la grotte renvoient déjà à des visages et à des formes signifiantes qui s'inscrivent dans le milieu naturel ("petrificaciones sin formas determinadas, que presentan masas deslumbrantes y figuras raras y carpichosas"). À la fin du roman, le souvenir est encore détenu par celui qui se voit désigné comme «le paysan de la terre rouge " ("sencillo labrador de la tierra roja" ${ }^{26}$ ), pour ensuite être repris par la voix narrative. Le détenteur et dépositaire de la mémoire est donc bien la terre, que celle-ci s'incarne dans les divers motifs du paysage, dans celui qui la travaille, ou dans celui qui la raconte. Parangon de l'Histoire, la terre est effectivement érigée en espace paradigmatique réinventé au service des discours mémoriels des Amériques sur lesquels il s'agit à présent de revenir. 


\section{"Paroles de nuit » et explicitation de la symbolique paysagère par Sab, héritier de Martina}

Consécutivement aux visions qu'il esquisse, le texte se charge donc très vite d'établir le récit, la parole d'une genèse. La vue des merveilles ou des stigmates du passé est toujours associée à la mise en place d'un récit historique.

La continuité entre espace du témoignage et récit est d'ailleurs instaurée sans équivoque. Au cours du voyage, c'est d'abord l'apparition de la mystérieuse lumière discontinue sur la colline qui permet l'insertion, l'enchâssement d'un nouveau récit dans le récit. À l'instar des autres éléments naturels, le motif lumineux est soumis à une mise en valeur particulière qui lui confère le statut d'amorce discursive puisqu'il devient l'objet principal du propos et prépare l'exploitation de l'allégorie de la caverne. Outre la mention du phénomène par la voix narrative, c'est la mise en scène de l'attention que lui portent chacun des personnages, avec notamment une nouvelle allusion à l'enthousiasme infantile (émotion associée à l'espace originel), et l'organisation de leur positionnement dans l'espace, autour d'un Sab conteur, qui permet cette mise en relief et laisse place à l'explicitation suivante :

Su merced no habrá olvidado a la vieja Martina, madre de uno de sus mayorales de Cubitas [...]. A esa vieja pues, a Martina es a quien he oído repetidas veces, referir misteriosamente e interrumpiéndose por momentos con exclamación de dolor y pronósticos siniestros de venganza divina, la muerte horrible y bárbara que, según ella, dieron los españoles al cacique Camagüey, señor de esta provincia; [...]. Camagüey tratado indignamente por los advenedizos, a quienes acogiera con generosa y franca hospitalidad, fue arrojado de la cumbre de esa gran loma y su cuerpo despedazado quedó insepulto sobre la tierra regada con su sangre. Desde entonces esta tierra tornóse roja en muchas leguas a la redonda, y el alma del desventurado cacique viene todas las noches a la loma fatal, en forma de luz, a anunciar a los descendientes de sus barbaros asesinos la venganza del cielo que tarde o temprano caerá sobre ellos. Arrebatada Martina en ciertos momentos por este furor de venganza, delira de un modo espantoso y osa pronunciar terribles vaticinios. - ¿Y cuáles son? - preguntó don Carlos con cierta curiosidad inquieta, que mostraba haber sospechado ya lo que preguntaba. Sab se turbó algún tanto pero dijo al final con voz baja y trémula: - En sus momentos de exaltación, señor, he oído gritar a la vieja india: "La tierra que fue regada con sangre una vez lo será aún otra : los descendientes de los opresores serán oprimidos, y los hombres negros serán los terribles vengadores de los hombres cobrizos". ${ }^{27}$

La restitution des mots de la légende de Martina par Sab l'esclave, assimilé au guide et au conteur, propose un récit à la fois mythique et prophétique, historique et prospectif. Établissant encore un continuum temporel sur un axe passé, présent et futur, le récit déborde tout cloisonnement. En réélaborant le discours de l'histoire de la Conquête de l'̂̂le, il fait écho aux effets de sens initialement produits par l'édifice symbolique dispersé dans la nature, et en fournit une explication. Dans ces contrées la terre est rouge, car maculée du sang du roi Camagüey, dont le corps délogé des hauteurs de sa colline par les premiers Espagnols, fut morcelé, "despedazado », nous dit significativement Sab. Un peu argileuses et minérales, les terres de la région de Cubitas sont effectivement teintées d'une couleur ocre. Ce qui est intéressant ce n'est pas la seule adéquation à cette réalité géologique du terrain, commune à plusieurs îles de la Caraïbe, mais l'exploitation poétique qui en est faite, par le truchement d'une parole qui est celle du récit fantastique ou du conte merveilleux. Ce dernier donne d'abord lieu à l'émergence de la figure 
massive, et à peine discernable, de la colline assimilée à un "géant aérien " qui projette l'énormité de son ombre. Cette forme à la fois immatérielle et massive annonce la présence de l'âme et du corps de la figure du vengeur. Le paysage-traces trouve ainsi son incarnation dans une figure fondatrice.

L'éclairage qui fait entrer dans un univers nocturne et inquiétant est également celui de la parole subversive du conteur et de l'imaginaire, dans les tropiques où la nuit tombe vite et où les bruits émergent. Durant ce voyage qui s'achève au crépuscule, c'est donc une " parole de nuit » qui surgit du texte romantique. On y retrouve un imaginaire de la nuit tropicale comme lieu d'expression et de libération de la parole, dans une mise en scène qui s'approche de ce que Ralph Ludwig expose à propos du conte créole :

Depuis le siècle de Voltaire et de Diderot, la lumière incarne la métaphore clé de la pensée analytique véhiculée par l'écriture. L’Europe a voulu «éclairer » l'Afrique "noire" et les Antilles par la colonisation et l'alphabétisation. La lumière du jour était réservée au travail, à la culture et à la langue officielles. [...] la nuit, au contraire, a toujours été le lieu de la parole créole. C'est au crépuscule que le conteur créole réunit son auditoire. C'est une fois la nuit tombée qu'on raconte la vie des ancêtres aux enfants, et c'est là encore qu'on fête leur mort au cours des veillées. La nuit, c'est l'univers du loisir, du plaisir sensuel et de l'insoumission à l'égard des restrictions de la journée. Sur le plan de la réflexion philosophique, prendre conscience de l'opacité revient à refuser le totalitarisme de la raison cartésienne, de la clarté. Intégrer l'opacité et le côté mythique de l'oral à l'analyse amène à relativiser cette dernière, en retraçant par là même l'itinéraire intellectuel des romantiques du XIXe siècle qui s'aperçurent que l'esprit du siècle des Lumières était incapable - à lui seul - de rendre compte de l'ensemble de la réalité humaine et de son avenir et qui découvrirent alors des genres littéraires proches de l'oral. C'est ainsi que la parole du conteur créole n'est parfois « pas claire » et que, dans la littérature antillaise, sur le plan de la stratégie de l'écriture, la causalité fait place à l'association. ${ }^{28}$

Dans la légende du cacique, la lumière devenue explicitement corps de ce dernier est révélatrice de l'entreprise d'établissement de la filiation explicite entre les Indiens et les esclaves noirs. Le nom de Camaguëbax, personnage du récit de Martina, qu'elle présente comme son ancêtre, signifierait d'ailleurs « fils de l'arbre ». Sachant que Martina est ellemême assimilée à l'arbre, cette possible signification confirme l'exploitation des symboles paysagers et de la matière, dans l'établissement des filiations multiples et à double-sens, notamment au travers de figures ancestrales, ou filiales, interchangeables. Il s'agit là d'un des aspects les plus novateurs de ce texte qui entre en rupture avec les cloisonnements génériques $\mathrm{du} \mathrm{XIX}^{\mathrm{e}}$ siècle séparant les écrits indianistes du roman de l'esclave, pour proposer une écriture de la complexité de la condition du sujet colonial et de son histoire. C'est en effet l'esclave mulato Sab qui relaie cette parole de Martina, supposément mestiza, détentrice de la légende. D'autres modalités de cette filiation apparaissent d'ailleurs dans la construction même de ces deux personnages "métis", qui ne mènent à aucune certitude, car leur phénotype reste mystérieux. Ils constituent tous deux des hérauts indistincts pour la refonte des identités et la mise en lumière de l'histoire des Antilles ${ }^{29}$. À la filiation discursive s'ajoute la filiation symbolique, par le dépassement de considérations typifiantes qui n'envisageraient que le seul phénotype, et par la position de Martina qui remplit la fonction de mère adoptive pour Sab. Ces fonctions du métissage sont ainsi à rattacher à l'esthétique du paysage-trace dans l'écriture de la mémoire.

Sab et Martina constituent les pièces maîtresses de ce qui tend à devenir un édifice polyphonique, un jeu de dispersion des voix brisant la logique du discours unique. Telle 
qu'elle se donne à « lire » dans l'économie générale du récit, la légende révèle que l'on confère finalement une place centrale à des paroles pourtant précautionneusement présentées comme marginales et problématiques. À l'instar de ce qui régit le choix de la forme fantastique, l'ambivalence du statut de cette parole est tout autant une stratégie qu'une esthétique, puisque les dires ne cesseront d'être rappelés et déclinés sous diverses formes. Ils « envahiront » ainsi la narration, à l'image de ce qu'évoque Ralph Ludwig:

L'auteur antillais d'aujourd'hui ne présente plus la réalité de son archipel comme le ferait une encyclopédie tropicale. Il associe le lecteur au mysticisme du vaudou, au rythme circulaire de la narration, au refus de réduire la réalité complexe à une formule analytique. Il répond en cela à un besoin du lecteur européen à la recherche de refuges mythiques..$^{30}$.

\section{Les variations du dire pour une rupture de la logique et de la linéarité cartésiennes}

Si le récit de Martina, tel qu'il est relayé par Sab, constitue un premier prolongement et un développement d'une voie/voix légendaire et fantastique qui fait émerger un mythe fondateur à Cuba, depuis une expérience originelle traumatique, l'histoire se prolongera dans un discours noyé par la multiplicité des paroles.

Pas plus qu'à la fonction mimétique de composants paysagers qui présupposeraient un référent extérieur absolu et une immédiateté du voir et du savoir, ni à celle d'une chronologie linéaire, l'histoire n'est ici nullement soumise à la logique de la rationalité, "delira de un modo espantoso" dit-on en parlant de Martina. C'est tout au long de l'épisode que l'on ne cesse d'ailleurs d'en souligner le caractère marginal, hétérodoxe, ou encore significativement sujet à caution par le colon :

Los naturalistas- les dijo [don Carlos] - os darían del fenómeno que estáis mirando una explicación menos divertida que la que os puede dar Sab, que frecuenta este camino y trata a todos los cubiteros. Él sin duda les habrá oído relaciones muy curiosas respecto a la luz que tanto os ha llamado la atención. ${ }^{31}$

La parole « extravagante $»^{32}$ tente également de s'affranchir du rapport au langage écrit (bien que dans les faits le récit légendaire soit restitué par le truchement d'un texte narratif intégré à la diégèse romantique), en ce qu'elle est multiforme ou encore formée autrement.

En jouant sur les mots, la voix narrative suggère d'ailleurs dès le début du voyage qu'elle prend une forme nouvelle: "Todos los viajeros se reunieron en torno a la linda criolla, y Sab les manifestó su plan de marcha. Iba, dijo, a conducirlos a Cubitas no por el camino real sino por una senda poco conocida, que aunque algo más dilatada les ofrecería puntos de vista más agradables." ${ }^{33}$ Sab, esclave et guide, fixe le parcours inhabituel de ce voyage initiatique qui est donc à la fois détour et retour dans l'espace devenu symbole des méandres d'une mémoire collective "mise sous verrous $»^{34}$. Détournement donc de l'itinéraire, "del camino real", c'est-à-dire de l'histoire officielle de la Conquête par la royauté espagnole. Le cheminement inédit pour accéder à cet espace mène à l'opposition "real" et "fantástico", laquelle revêt un double sens, afin de signifier que cette histoire correspond également à un déplacement du point de vue logique et esthétique.

41 C'est immédiatement que le dire est placé sous le signe de l'altérité puisqu'il est d'abord restitution «orale » d'une parole autre. Dans les grottes, il prend ensuite la forme du signe peint qui rejoint les traditions transmises par les habitants de Cubitas et Sab, alors significativement appelé Cicéron. Notons la référence évidente à l'art oratoire du 
personnage de la République romaine qui octroie à l'esclave toute la légitimité du statut d'orateur, de véritable détenteur d'une histoire à transmettre. On remarquera l'emploi d'une typographie distincte pour la retranscription de cette appellation (des italiques dans le texte de l'édition citée), qui révèle l'existence de la mise en relief initialement choisie par Gómez de Avellaneda elle-même : "Don Carlos de B... y su familia, llevando a Sab por cicerone, emprendieron, al día siguiente a su llegada a Cubitas, la visita de esas grutas." ${ }^{35}$

42 Enfin, le récit pourra ne devenir qu'un mot, qu'un nom sur les murs: "Las paredes estaban llenas con los nombres de los visitadores de la grutas, pero la compañía no pudo dejar de manifestar la mayor sorpresa al ver el nombre de Carlota entre ellos, no habiendo ésta visitado hasta entonces aquellos sitios." ${ }^{\prime 36}$ Cette scène sert d'abord à suggérer que c'est Sab, l'amoureux éploré, qui a inscrit le nom de Carlota, mais elle révèle également à quel point l'inscription du nom marque un passage. Dans la pierre de la grotte, le mot unique est la marque d'un récit alternatif qui s'associe aux autres formes de récits visuels ou oraux, intimement liés au paysage. Paysage et parole ne font alors plus qu'un. Le paysage est récit et le récit est paysage, dans ce qui s'apparente à une véritable assimilation. Notons la structure et le lexique de la phrase suivante, au sein de la description des grottes :

los naturales hacen notar [...] pinturas bizarras designadas en las paredes con tintes de vivísimos e imborrables colores, que aseguran ser obra de los indios, y mil tradiciones maravillosas prestan cierto encanto a aquellos subterráneos desconocidos; que realizando las fabulosas descripciones de los poetas recuerdan los misteriosos placeres de las hadas. ${ }^{37}$

43 Malgré la ponctuation, dans le mouvement de cette longue phrase (que nous avons pris la liberté de tronquer), et du fait de l'impersonnalité du participe présent, ce sont l'œuvre des peintures comme les traditions qui peuvent être associées aux discours descriptifs des poètes. On ne saurait alors dire si le discours se place en amont ou en aval du signe visuel. Par ailleurs, l'emploi du participe "designadas" fait l'objet du commentaire suivant de José Servera, l'éditeur du texte dans la version utilisée pour cette étude : “designadas. No parece un vocablo muy apropiado, pues significa señalar, destinar, indicar. Mary Cruz propone diseñadas (edición de Sab, p. 297)"38. Qu'il s'agisse d'une « erreur » de l'écrivaine ou d'un choix conscient, il est intéressant de remarquer que, de facto, en créant une redondance (on nous signifie, on nous signale qu'il y a un signe), cet emploi participe d'un effet de sens qui entérine l'assimilation du dessin au verbe ${ }^{39}$. Le trait signale, dit ou encore montre les peintures (fait-il partie de celles-ci ou alors est-il placé à côté ?). Cellesci sont ensuite immédiatement associées à un récit. Le trait serait donc le signe qui invite au discours qu'est la peinture elle-même. Il s'agit encore d'une stratégie de mise en évidence devant le voyageur, qui doit voir, dans tout signe visuel, le paysage comme ce qui est intrinsèquement porteur d'histoire.

Conjointement, les effets de répétition et d'éclatement se multiplient. Rappelons que les deux temps du propos restitués par Sab (l'événement originel puis la prophétie), sont déjà eux-mêmes entrecoupés du commentaire suivant qui, par l'usage du présent, suggère la récurrence du dire: "Arrebatada Martina en ciertos momentos por este furor de venganza, delira de un modo espantoso y osa pronunciar terribles vaticinios." ${ }^{40}$ On remarquera d'ailleurs que son contenu prophétique tend à construire une visualisation, une mise en scène déjà fondée sur les événements de la rupture (la Conquête), puis d'une reprise, dans la répétition et la variation (la vengeance). À de très nombreuses reprises, la narration, que ce soit dans le propos des notes, de la voix narrative ou des personnages, 
souligne le caractère redondant et opaque des paroles de Martina, relayées par tous les habitants de Cubitas : "adoptamos por ahora la opinión de los cubiteros, y explicaremos el fenómeno, en la continuación de la historia, tal cual nos ha sido referido y explicado más de una vez" ; également "oyéndola referir sus sempiternos cuentos de vampiros"; "A esa vieja pues, es a quien he oído, repetidas veces, referir misteriosamente e interrumpiéndose por momentos de exclamación y dolor y pronósticos siniestros"; ou enfin "Martina aprovechó la ocasión que le dieron algunas preguntas de Carlota, para repetir los maravillosos cuentos que ya mil veces había contado" ${ }^{41}$. Sans toutefois répéter les détails de la substance du discours historique et prophétique de l'Indienne (tel que cité une première fois par Sab), la narration se charge donc d'en proposer régulièrement des rappels allusifs, notamment au cours du dîner final chez Martina qui s'apparente à une veillée. L'allusion à ces contes merveilleux stimulés par l'imagination enfantine réaffirme toujours l'existence d'une parole excentrée et excentrique, dans une maison déjà fondue dans le paysage par la brûlure du bois et assimilée à la caverne.

En d'autres termes, si, par essence, la parole de Martina est répétitive, la narration ellemême la répète, puisque son discours est repris dans plusieurs passages. La parole est ainsi toujours relayée, rappelée, reformulée. Sur elle on glose. Ces commentaires constituent autant de variations d'un même dire. Et plus largement, ce sont tous les propos autour de Martina (sa légende, comme sa personne, son lieu) qui seront redondants. Ces deux aspects que sont la discontinuité et la répétition ne sont donc pas sans " altérer » quelque peu la logique des codes de la narration écrite elle-même. Avec ces jeux d'échos et de correspondances permanents, les paroles martiniennes sont bien celles de la rupture et de la variation.

Elles sont donc reliées à l'oralité, non seulement par l'atmosphère des veillées, par les dénominations qu'on leur donne (tantôt associée à la prophétie, tantôt au délire, tantôt aux explications drôles ou aux contes merveilleux), par leur contenu thématique qui les rapproche du mythe (forme de l'attente et jaillissement particulier de l'évènement, comme le rappelle Louis Marin $^{42}$ ) mais aussi par leur structure logique, ou plutôt non logique, et leur rythme. Ces éléments caractéristiques de l'oralité sont donc visibles audelà de l'apparition thématique de leur mode d'émergence (parole prononcée par les personnages), puisqu'on les retrouve dans certains des détails mêmes de leur «mode de production ».

Par conséquent, la parole devient collective et plurielle (on notera l'usage récurrent de la forme plurielle pour les dénominations de "cuentos" "vaticinios", etc. qui la démultiplient). Non seulement écoutée de tous, et temps du rassemblement, elle fait toujours parler. Dès le départ, l'épigraphe au contenu ambivalent ne dit pas autre chose. Cette citation de Guy Mannering de Walter Scott reprend en effet certaines des caractéristiques précitées. On y trouve déjà commentaire sur la forme d'un discours « non organisé » mais non moins légitime : "La mezcla de extravagancia y de entusiasmo que reinaba en sus discursos rara vez dejaba de producir la más viva impresión en aquellos que la escuchaban. Sus palabras con frecuencia entrecortadas eran, empero, demasiado claras e inteligibles para que pudiese sospechársele en un verdadero estado de locura." ${ }^{43}$ La représentation du discours oral et légendaire comme phénomène non structuré et non logique n'est qu'une manière d'atténuer l'impact du propos subversif.

Au-delà de leur fonction « intellectuelle » et de l'effet caution qu'elles produisent, dans leur ensemble, les épigraphes ${ }^{44}$, dont l'une des thématiques principales est la parole, constituent l'un des instruments qui permettent tout autant d'introduire la répétition 
que la variation. Annonçant et rappelant, elles revêtent une «fonction sensible » au service de la polyphonie, créant ainsi de nombreux échos et démultipliant les paroles.

Ces éléments de forme qui font partie de la stratégie exposée précédemment, visant à minorer le discours de Martina sans jamais le discréditer complètement, ont donc également des implications sur l'ensemble de la logique discursive de la narration ellemême. En effet, au-delà des seuls chapitres IX et X, dans son entier, le texte s'écartèle déjà très clairement entre épigraphes, narration, dialogue, notes et ici conte, mythe ou légende, pour devenir un magma textuel, un récit polymorphe. De cette polymorphie et polyphonie, qui ne permettent finalement d'établir aucune hiérarchie entre les dires, émergent les effets de l'ambivalence, mais aussi de la redondance et de l'amplification. Si nous avions souligné que c'était l'Indienne qui constituait le principal foyer de la parole légendaire, marginale, fantasque ou encore fantastique, force est de constater que la narration s'en imprègne, rompant ainsi la linéarité inhérente à l'écriture. Le roman luimême imite sans cesse ce qu'il présente thématiquement comme un discours de la répétition. Il est pleinement traversé par la redondance, il la pratique. Dans un mouvement centripète, il oblige à des allers et des retours. Il reprend ainsi la structure répétitive à son compte pour l'étendre. Prenons pour seul exemple la note concernant Cubitas : "Las cuevas de Cubitas son una obra admirable de la naturaleza, y dignas de ser visitadas. Más adelante hablaremos de ellas con alguna más extensión." ${ }^{45}$ Dès le début du roman, cette note cherche bien à attirer l'attention du lecteur sur cet univers, dans une formulation qui sera ensuite presque reprise terme à terme dans le corps du récit, au début du chapitre XI : "Las cuevas de Cubitas son ciertamente obra admirable de la naturaleza, que muchos viajeros han visitado con curiosidad e interés y que los naturales del país admiran con una especie de fanatismo." ${ }^{46}$

La narration se voit ainsi rythmée et scandée à partir de l'événement du discours légendaire de Martina, modulé à loisir. Le texte éclaté marque la brisure de la parole, comme la démultiplication et la libération de celle-ci. Toutes ces voix qui entrent en résonance disent l'expérience de la fragmentation et du déchirement ainsi que l'ambivalence d'une réalité à la fois une et plurielle. Plus que la visibilité d'une parole orale mise en scène de manière artificielle, ce qui revêt à nouveau un caractère novateur et fécond, c'est cette modalité presque imperceptible et pourtant fondamentale de l'écriture avellanédine. Celle-ci vient à assouplir le carcan habituel dans lequel se fixe la parole écrite de la narration romantique qui ne parvient souvent qu'à entretenir avec l'oralité un rapport thématique et folklorisant. Apparemment complètement livré à luimême, dans ce «maelström de signifiés » que constitue le roman, le lecteur semble donc se trouver face à de l'indécidable, à l'image de ce que met en exergue le texte de l'Éloge de la créolité :

Notre personnalité culturelle porte tout à la fois les stigmates de cet univers et les témoignages de sa négation. Nous nous sommes forgés dans l'acceptation et le refus, donc dans le questionnement permanent, en toute familiarité avec les ambigüités les plus complexes, hors de toutes réductions, de toute pureté, de tout appauvrissement. Notre histoire est une tresse d'histoires. Nous avons goûté à toutes les langues, à toutes les parlures. [...]. La Créolité c'est « le monde diffracté mais recomposé », un maelström de signifiés dans un seul signifiant : une Totalité. ${ }^{47}$ 


\section{Conclusion : u-topie et impossibles ancrages}

51 Étrange topie compensatoire, cachée sous ou dans la topie, Cubitas n'est-il finalement pas un espace u-topique ? Dans son sens premier et littéral l'utopie, « oú-topia », est un nonlieu $^{48}$. La refonte de ce lieu s'inscrit bien dans cette tension contradictoire au sein d'une articulation particulière entre le discours sur l'espace et les formes de son énonciation. Le passage par les figures et l'usage du symbolique permettent une recréation ambivalente qui remplit effectivement un vide historique.

Narrant un voyage dont l'itinéraire est approuvé par tous les personnages ${ }^{49}$, le roman et les paysages qu'il dresse constituent une sorte de compromis entre l'histoire officielle de la Conquête et les possibles histoires de l'esclave et de l'Indien, pour une mémoire collective. D'un point de vue idéologique, Sab se caractérise dans son ensemble par la forte ambivalence de son propos. On y relève le caractère subversif, plus ou moins caché, du discours antiesclavagiste et de la réécriture historique. L'œuvre se trouve pourtant également prise dans l'établissement d'une forme de nostalgie pour des temps révolus, et d'idéalisation de la figure du doux colon face à la figure du capitaliste E. Otway. Les « airs salutaires $\aleph^{50}$ de Cubitas, tentative de recréation d'une origine perdue, objet d'une passion fanatique, constituent alors un espace de compromis ou de franche subversion, selon le degré de lecture des discours qui y apparaissent, dans une irréductible et inépuisable tension.

53 Toutefois, la pensée nouvelle de toute l'articulation entre la construction des personnages mouvants, du paysage/espace et des modalités multiples des récits confèrent à l'œuvre, dans son contexte d'émergence, un caractère résolument novateur. Nous l'avons vu, l'établissement de cet espace mémoriel, construit sur le plan symbolique, sous une certaine forme figurative mais aussi partiellement indéfinie, où se nichent plusieurs temporalités et une grande pluralité discursive, présente de nombreuses accointances avec celui des paysages de l'exhumation qui apparaissent dans nombre de poétiques caribéennes et/ou hispano-américaines postérieures ${ }^{51}$, où l'incertain de la parole, l'opacité, le détour et la répétition deviennent les éléments d'un nouveau langage de résistance et d'une contre-poétique possible. En effet, comment ne pas établir, en dernier lieu, des liens directs entre ce roman et le passage suivant du texte programmatique de l' Éloge de la créolité :

Les paysages sont les seuls à inscrire, à leur façon non anthropomorphe, un peu de notre tragédie, de notre vouloir exister. Si bien que notre histoire (nos histoires) n'est pas totalement accessible aux historiens. Leur méthodologie ne leur donne accès qu'à la Chronique coloniale. Notre Chronique est en dessous les dates, dessous les faits répertoriés: nous sommes Paroles sous l'écriture. Seule la connaissance poétique, la connaissance romanesque, la connaissance littéraire, bref, la connaissance artistique, pourra nous déceler, nous percevoir, nous ramener évanescents aux réanimations de la conscience. Appliquée à nos histoires (à cette mémoire-sable voltigée dans le paysage, dans la terre, dans des fragments de cerveaux de vieux-nègres, toute en richesse émotionnelle, en sensations, en intuitions...) la vision intérieure et l'acceptation de notre créolité nous permettront d'investir ces zones impénétrables du silence où le cri s'est dilué..$^{52}$

L'u-topie serait donc ici celle de recréer des espaces pour signifier l'impossible ancrage lié à la condition du sujet colonial, à rattacher, d'une part à la condition également problématique du sujet féminin qui ne trouve pas d'espace, mais aussi, à la logique 
d'ensemble des cartographies américaines de l'auteure. Il est en effet significatif que d'autres modalités de la dissolution des lieux apparaissent dans les récits que Gómez de Avellaneda a choisi d'ancrer dans les Amériques, au moment de réécrire les histoires de ces dernières. La réécriture fictionnelle qui émerge de ces nouvelles géographies imaginaires $^{53}$ mène alors à des situations de pure paratopie $e^{54}$. Au fil de leurs errances, les personnages sans résidence fixe se trouvent toujours pris dans la relation problématique entre l'Espagne et ses colonies.

\section{NOTES}

1. Jean Bernabé, Patrick Chamoiseau, et Raphaël Confiant, Éloge de la créolité, Paris, Gallimard, 1990, p. 36-38.

2. Édouard, Glissant, Le Discours antillais, [1981], Paris, Gallimard, 1997, p. 32.

3. On peut établir une typologie des géographies imaginaires (au sens d'Edward Said, voir L'Orientalisme, [1971], Paris, Seuil, 1997) américaines créées par l'auteure dans ses récits (concrètement Cuba, le Mexique et la Colombie). D'une part, apparaît le paysage sublime ou pittoresque, d'autre part figure ce que nous avons appelé le lieu-patrimoine qui cumule une fonction anthropologique, celle de l'ethnotexte (selon la définition de Valérie MagdelaineAndrianjafitrimo, "Ethnotexte" et intertextualité: la mise en scène des représentations culturelles dans les romans "ethnographiques" ", Univers créoles 4, V. Magdelaine et Carpanin Marimoutou (éds), Paris, Anthropos, 2004, p. 93-145), proche de l'écriture costumbrista, et une vocation mémorielle. Ces deux types de lieux contribuent à fixer une série d'identités et d'images, non exemptes d'un certain essentialisme et d'une teinte d'exotisme, bien que cette notion soit à nuancer. On y retrouve ainsi une visée laudative, (re)présentative et/ou informative dans la mise en scène du territoire. Ces lieux de mémoire (au sens de Pierre Nora, Les lieux de mémoire, Paris, Gallimard, "La République », Tome 1, 1984, "La Nation », tome 2, 1986, "Les France ", tome 3,1992), recrées et idéalisés depuis la distance, participent d'une cartographie de la nostalgie, ancrée dans un référentiel très détaillé et concret et révèlent le maniement d'un vocabulaire spécifique.

4. La différenciation qu'effectue Michel De Certeau entre le lieu, statique, et l'espace, en mouvement, est éclairante pour l'analyse du fonctionnement des textes et des discours avellanédins, voir L'invention du quotidien, « Arts de faire », Paris, Éditions Gallimard, 1990.

5. D'après l'expression de Michel de Certeau, Ibid., p. 170-171.

6. D'après le concept d'Édouard Glissant, op. cit., p. 32.

7. Gertrudis, Gómez de Avellaneda, Sab, José Servera ed., Madrid, Ediciones Cátedra, 2001, p. 163.

8. Ibid., p. 165-166.

9. À ce sujet, voir notamment l'ouvrage de Charles Le Blanc, Laurent Margentin, Olivier Schefer, La forme poétique du monde, Anthologie du romantisme allemand, Paris, José Corti, 2003, p. 35.

10. Voir ce que rappelle José Servera dans Gertrudis, Gómez de Avellaneda, Sab, op. cit., p. 48-49.

11. Élisabeth Pluton fait le choix de traduire le premier terme par " palmier-balai » et le second par «ficus ». Pour notre part, afin de mieux insister sur l'existence de ces réalités propres telles qu'elles sont spécifiées et reconstruites par l'imaginaire avellanédin, nous avons choisi de ne pas tenter de les traduire dans le corps de l'analyse. Pour le terme de "guano", visant lui-même à expliquer ce qu'est un "yuraguano", É. Pluton propose celui de palmier. Voir Gertrudis, Gómez de 
Avellaneda, Sab, Traduction d'élisabeth Pluton et présentation de Frank Estelmann, Paris, L'Harmattan, 2010, p. 71.

12. Gertrudis, Gómez de Avellaneda, Sab, Edición de José Servera, op. cit., p. 165, note $\mathrm{n}^{\circ} 94$ et note $\mathrm{n}^{\circ} 95$.

13. Ibid., p. 173-175.

14. Olivier Schefer rappelle que les nombreuses représentations romantiques de la brillance de la nuit (et notamment les grottes, les phares, les torches, les ombres, etc.), dans les textes et dans la peinture, sont le lieu de la mise à jour de ce qui a été vu dans l'obscurité. Ces représentations sont intimement liées à la conception d'une imagination comme puissance de connaissance excédant justement les limites de la représentation. Ces motifs montrent que l'homme rêve pour ne pas cesser de voir, que la nuit (intérieure ou extérieure) est le lieu de la clairvoyance (in "L'imagination nocturne du romantisme », communication d'O. Schefer, 15 mars 2013, lors d'une journée d'étude sur "Romantisme, romantismes", à l'auditorium du Musée d'Orsay, dans le cadre de la présentation de l'exposition L'ange du bizarre : le romantisme noir de Goya à Max Ernst).

15. Gertrudis, Gómez de Avellaneda, Sab, José Servera ed., op. cit., p. 180 et 181.

16. Ibid., p. 183.

17. Ibid., p. 263.

18. Renvoyant à l'ancrage impossible, le non-lieu est entendu au sens que lui confère Marc Augé, qui associe ce phénomène à la coexistence de plusieurs mondes et à l'absence d'identité singulière. Ce phénomène serait propre à la postmodernité ou à la surmodernité, d'après lui. Voir Non-lieux, Introduction à une anthropologie de la surmodernité, Paris, Seuil, La librairie $\mathrm{du} \mathrm{xx}^{\mathrm{e}}$ siècle, 1992, p. 117-118 et p. 127.

19. L'image finale de la fantomatique Martina est la suivante: "visión misteriosa que posteriormente se dejó ver todas las noches por espacio de tres meses, en el mismo lugar", Gertrudis Gómez de Avellaneda, op. cit., p. 274.

20. Ibid., p. 177.

21. Ibid., p. 170.

22. Ibid., p. 182.

23. Certes, dans l'économie du roman, c'est-à-dire dans le cadre de l'histoire contée depuis l' incipit, la voix narrative se charge de définir la durée précise du séjour (trois jours), dans une appréhension du temps cohérente avec la chronologie d'ensemble, mais aucune date ou périodisation précise ne figure dans l'épisode. De manière générale, aucune date n'est d'ailleurs clairement mentionnée dans le roman dont la chronologie reste floue. On insiste davantage sur les durées. Voir Ibid., p. 189, p. 191 et p. 256, ainsi que les propos de Mary Cruz in Gertrudis Gómez de Avellaneda, Sab, La Habana, Editorial arte y literatura, 1976, p. 287, note $n^{\circ} 2$.

24. Édouard, Glissant, op. cit., p. 473-474.

25. Voir Gertrudis, Gómez de Avellaneda, Sab, José Servera ed., op. cit., p. 246.

26. Ibid., p. 273-274.

27. Ibid., p. 167-168.

28. Ralph Ludwig, Écrire la parole de nuit, La nouvelle littérature antillaise, Paris, Éditions Gallimard, 1994, p. 18-19. Le propos marquant la rupture entre les Lumières et le Romantisme est toutefois à nuancer.

29. Voir notre étude sur les visions et fonctions du métissage dans cette œuvre de l'auteure : Marie, Joséphine, "Perspectivas poscoloniales en Gertrudis Gómez de Avellaneda: intuición poética del mestizaje”, Revolución y Cultura, La Habana, № 4, oct-déc 2014, p. 2-6.

30. Ralph Ludwig, op. cit., p. 18-19.

31. Gertrudis, Gómez de Avellaneda, Sab, op. cit., p. 167.

32. Expression tirée de l'épigraphe qui ouvre le chapitre $X$ narrant la visite des voyageurs chez Martina, une citation de Walter Scott qui introduit la figure de ce personnage particulier, Ibid., p. 173. 
33. Ibid., p. 164.

34. Expression de Jean Bernabé, Patrick Chamoiseau et Raphaël Confiant, op. cit., p. 14.

35. Ibid., p. 177.

36. Ibid., p. 175-176.

37. Ibid., p. 174.

38. Ibid., p. 174, note $\mathrm{n}^{\circ} 107$.

39. Une première occurrence du mot employé dans ce sens, et déjà considérée comme erronée par Mary Cruz, apparaît dans le roman, dans un tout autre contexte. Voir Ibid., p. 136, note n 54 .

40. Ibid., p. 168.

41. Ibid., p. 167,168 et 184 .

42. Voir Louis, Marin, Utopiques : jeux d'espaces, Paris, Éditions de Minuit, 1989.

43. Gertrudis, Gómez de Avellaneda, Sab, op. cit., p. 173.

44. Pour l'étude des épigraphes nous rappelons les précieux travaux d'Antoine Compagnon, La seconde main, ou le travail de la citation, Paris, Seuil, 1979. Gérard Genette, Seuils, Paris, Seuil, 1987.

45. Ibid., p. 128.

46. Ibid., p. 173. La variation et l'effet de répétition et d'amplification de certains propos n'est d'ailleurs pas l'apanage de ce texte. On les retrouve dans un autre roman de l'auteure, Guatimozín, où les notes se répètent et se complètent souvent. Le discours soulignera d'ailleurs lui-même qu'il se répète.

47. Jean Bernabé, Patrick Chamoiseau, Raphaël Confiant, op. cit., p. 27.

48. D'après la définition de Louis Marin, Utopiques : jeux d'espaces, Paris, op. cit.

49. Voir Gertrudis, Gómez de Avellaneda, Sab, op. cit., p. 164.

50. Ibid., p. 272.

51. Voir par exemple ce que dit Édouard Glissant, à propos de Jacques Stephen Alexis, de Gabriel García Márquez ou encore d'Agustín Cárdenas, ainsi que sa perspective critique d'ensemble, qui fait de son texte un point de référence éclairant auquel confronter nos narrations romantiques, op. cit., p. 343, p. 471-472, p. 769-773, et plus généralement, p. 460-485.

52. Jean Bernabé, Patrick Chamoiseau, et Raphaël Confiant, op. cit., p. 36-38.

53. Au sens d'Edward Said, voir L'Orientalisme, op. cit.

54. Voir Dominique, Maingueneau, Le discours littéraire: Paratopie et scène d'énonciation, Paris, Armand Colin, 2004.

\section{RÉSUMÉS}

Le roman $S a b$ entend réélaborer une mémoire par le paysage, lequel excède le geste de réappropriation symbolique d'un patrimoine nommé, décrit, circonscrit et valorisé par l'auteur romantique natif. Avec la recomposition non mimétique du paysage-trace (Glissant) à Cubitas, Gertrudis Gómez de Avellaneda fait du récit littéraire l'endroit de la compréhension et de l'expression d'une histoire antillaise brisée et occultée. Les lieux, qui ne sont plus des marqueurs d'identités, disparaissent au profit de la mouvance de divers espace-temps et d'un éclatement de paroles multiformes. Ces marques $d u$ refus $d u$ "grand récit " à l'occidentale révèlent une intuition de la postmodernité et de certains paradigmes postcoloniaux.

La novela Sab propone une reelaboración de la memoria por el paisaje, el cual supera el afán de reapropiación simbólica de un patrimonio nombrado, descrito, circundado y valorado por el 
autor romántico nativo. Con la recomposición no mimética del paisaje-huella (Glissant) en Cubitas, Gertrudis Gómez de Avellaneda convierte el relato literario en la herramienta de la comprensión y expresión de una historia antillana rota y ocultada. Los lugares, que ya no marcan las identidades, dejan paso al movimiento de varios espacios y temporalidades y a un estallido de palabras polifacéticas. Con aquellas señales del rechazo del "gran relato" occidental surge una intuición de la posmodernidad y de algunos paradigmas poscoloniales.

INDEX

Palabras claves : Avellaneda (Gómez de), memoria colonial, paisaje, no-lugar, polifonía Mots-clés : Avellaneda (Gómez de), mémoire coloniale, paysage, non-lieu, polyphonie

\section{AUTEUR}

\section{JOSÉPHINE MARIE}

Université Paris-Est Marne-la-Vallée (LISAA, EA4120) 